\title{
ASSISTING INTERRUPTION RECOVERY IN SUPERVISORY CONTROL OF MULTIPLE UAVS
}

\author{
Stacey D. Scott ${ }^{1}$, Stéphane Mercier ${ }^{2}$, M. L. Cummings ${ }^{1}$, Enlie Wang ${ }^{1}$ \\ ${ }^{1}$ Massachusetts Institute of Technology, Cambridge, MA, USA \\ ${ }^{2}$ École Nationale Supérieure des Telecommunications de Bretagne, Brest, France
}

Performance degradation due to interruptions is a critical issue, particularly when people are supervising highly autonomous systems in time and safety critical environments. Previous research in the development of automated support to help supervisory control operators resume task activities after an interruption has had limited success. This paper describes two new interruption recovery approaches that attempt to mitigate the disadvantages of previous approaches. In particular, this paper describes the design and initial investigation of a prototype assistive interface developed to support interruption recovery for supervisory control of multiple unmanned aerial vehicles (UAVs). These two 'replay'-type interruption recovery approaches enable increased user-control of the event discovery process and provide event 'bookmarks' to highlight emergent system events. The findings from this initial study provide several recommendations for the future design of interruption assistance tools for human supervisory control tasks.

\section{INTRODUCTION}

Interruptions are common in many work environments and have been shown to negatively impact task performance (Czerwinski et al., 2000; Loukopoulos et al., 2001; McFarlane, 1999). The time pressures of many supervisory control tasks make them especially vulnerable to the negative affects of interruptions (Bailey et al., 2001). For example, supervisory control of multiple unmanned aerial vehicles (UAVs) is a particularly time-critical, cognitively demanding task, which requires an operator to constantly monitor and update the progress of several vehicles during rapidly changing battlefield conditions. If a critical event occurs (e.g., a UAV flies into a hazardous area) while the operator is distracted, after the interruption the operator must first recognize the event occurrence, decide its potential impact, and finally take action. In the meantime, the performance of the UAVs will likely deteriorate while the operator is determining an appropriate course of action (Cummings \& Mitchell, accepted). To address this issue, this research is aimed at developing interruption recovery aids for supervisory control of multiple, independent UAVs. This paper describes the design and initial investigation of a prototype assistive interface developed to explore two potential interruption recovery approaches.

\section{ASSISTING INTERRUPTION RECOVERY}

Many approaches to assisting interruption recovery have focused on helping the user recall what they were doing before the interruption (e.g., Altmann \& Trafton, 2004). However, interruption recovery in a supervisory control task depends on understanding what changes have occurred in the system under control, which may or not be related to previous user actions. St. John, et al. (2005) have investigated interruption recovery in dynamic monitoring activities which represent a critical component of many supervisory control tasks. Their research showed that providing a textual event history list was more effective than a video replay tool in helping people resume their task. Their findings also showed that video replay typically led to slower interruption recovery. These findings, however, may have been influenced by limitations inherent to the design of their replay tool. For instance, their replay tool did not highlight any particular events, nor enable the users to control which events were replayed. Thus, operators had to spend considerable time watching the video replay to learn of past events. In contrast, the event history list provided a concise summary of critical events. A potential limitation of the event history list approach, though, is that when graphical events are described textually in a list, costly cognitive processing is required to relate the textual information to on-screen graphical components.

To explore the possibility of combining the benefits of the previous approaches, while mitigating their disadvantages, we developed a new interruption recovery assistance display, called the Interruption Assistance Interface. The main components of this display included an interactive event timeline that visually summarized past events using iconic bookmarks and a replay window for displaying past events in their appropriate context. To explore ways of improving the discovery of past events, two interaction methods for viewing events were developed: bookmarked and animated replay. In bookmarked replay, when an event bookmark was selected, the replay window displayed the system state at the time the event occurred. In animated replay, a particular sequence of events could be replayed in the replay window.

\section{The UAV Supervision and Replanning Task}

The representative task involved supervising and updating the mission plans for four independent homogeneous UAVs. There were two primary displays for this task: a tactical display and a decision support display (Figure 1). The tactical display provided a vehicle interaction panel and a situation map depicted the UAVs, scheduled routes, ground targets, and threat areas. The decision support display provided a chat window, UAV status information, and a decision support window that provided UAV mission timelines and replanning recommendations. One interruption recovery-related feature 

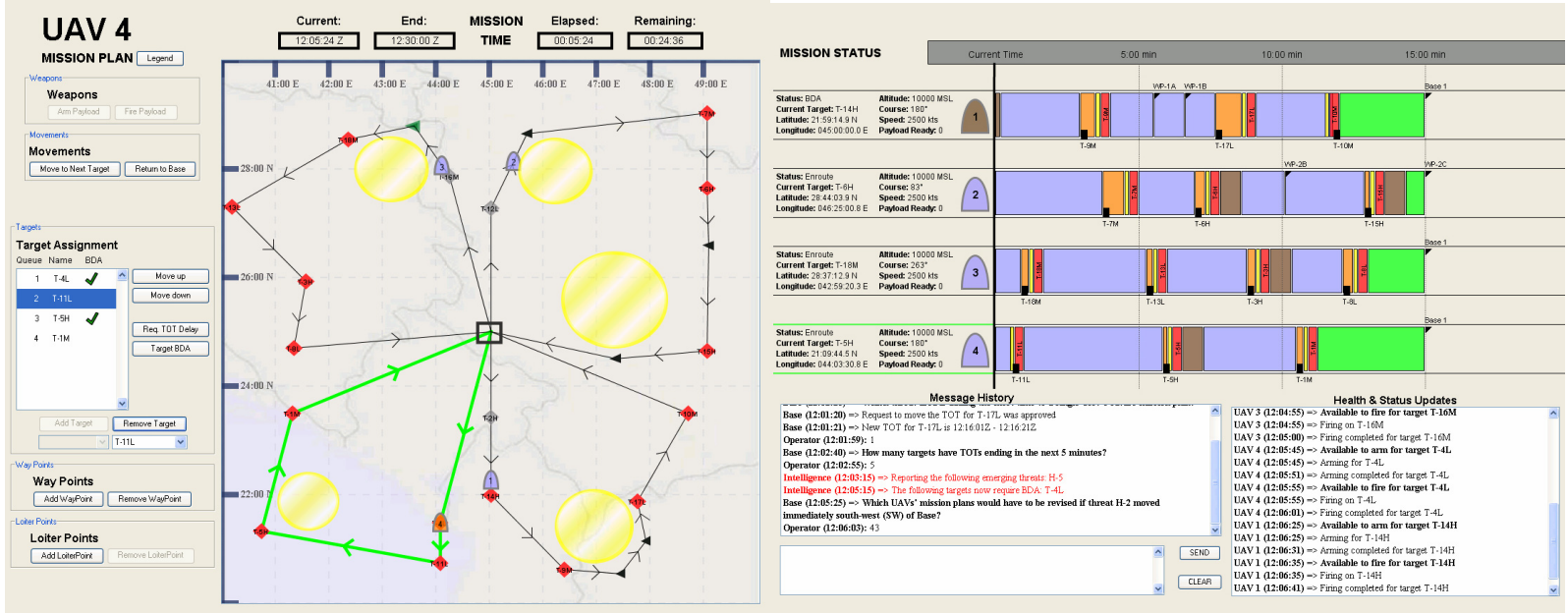

Figure 1. The tactical (left) and decision support (right) displays of the multiple UAV supervision and replanning task.

of the original display was the message history available in the chat window. Critical event messages were highlighted in red and remained available for viewing throughout the mission.

\section{Interruption Assistance Interface}

The Interruption Assistance Interface, a separate display (Figure 2), consisted of a replay window, an event timeline, and a set of animation controls (active only in animated replay). The replay window displayed historical views of the situation map from the tactical display of the UAV supervision and replanning task. This interface was designed as an always-available, peripheral display which continually updated as new events occurred in the main task environment. In order to understand the impact of the two interaction methods for viewing past events in the replay window on interruption recovery, two variations of the Interruption Assistance Interface were used in the experiment: one that provided bookmarked assistance and one that provided animated assistance. These variations are described below.

Bookmarked Assistance. In the bookmarked replay interface, selecting an event bookmark from the event timeline caused the replay window to display the state of the tactical map when that event occurred. The events bookmarked in the event timeline included: UAV status change (e.g., loiter entry, arming weapons, or changing routes) and the emergence or disappearance of a threat area or target (Figure 3). UAV status event bookmarks were displayed in the row associated with the relevant UAV. Threat area and target event bookmarks were displayed in the bottom two rows of the event timeline. A green horizontal bar showed the elapsed time. A blue vertical line on this time bar indicated the time of the events being displayed in the replay window. Finally, the object corresponding to the current event was highlighted in green in the replay window (Figure 2).

Animated Assistance. In the animated replay interface, users could view an accelerated ( $\sim 10 \mathrm{x}$ real-time speed) animated sequence of events by selecting a desired time window in the event timeline. The replay could then be controlled via the animation controls. The selected time window was indicated by a blue line displayed below the elapsed time bar (Figure 4). This interface also allowed users to view system events discretely by selecting individual bookmarks.

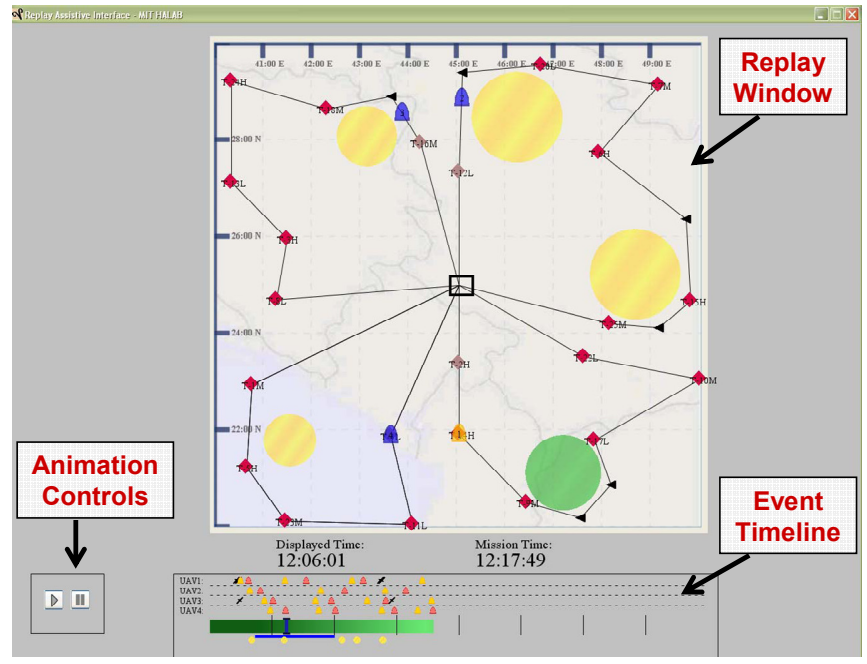

Figure 2. The Interruption Assistance Interface.

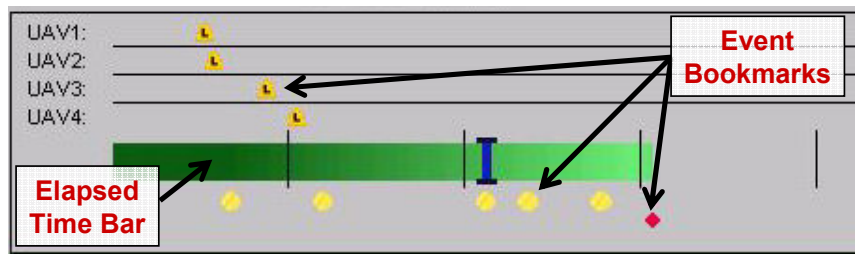

Figure 3. The event timeline showing UAV status, threat and target event bookmarks and the elapsed time bar.

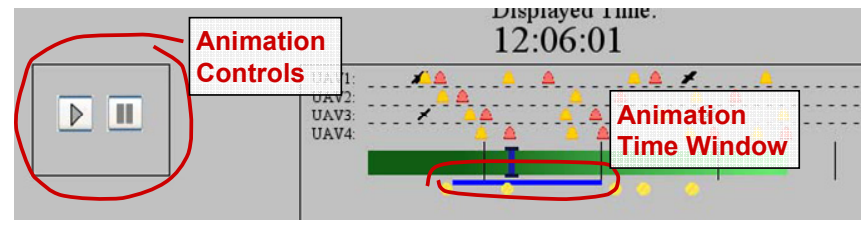

Figure 4. The animation controls and animation time window indicator. 


\section{EXPLORATORY STUDY}

\section{Participants and Apparatus}

Nine university students participated in this study. The primary experimental task was performed on a three-display, Windows-based workstation. In all sessions, the tactical and decision support displays were located on the central and right monitors, respectively. In the sessions with replay assistance, the Interruption Assistance Interface was located on the left monitor. The interruption task was performed on a standard Windows-based workstation, located in an adjacent room.

\section{Experimental Design}

The experiment was a 3 (Assistance Type) x 2 (Decision Difficulty) mixed design, with repeated measures on the Decision Difficulty factor. The three levels of the Assistance Type factor included: no assistance, bookmarked assistance, and animated assistance, as described previously. The two levels of the Decision Difficulty factor included: simple and complex. This factor related to the type of decision participants faced when they returned to the primary task. With a simple decision, only one possible action would address the current situation. With a complex decision, several possible actions would address the immediate situation, but based on past events there was one option that best met the mission criteria. For example, one complex decision situation included the emergence of a threat area on a UAV flight route and thus the affected UAV had to be re-routed around the threat area. However, during the interruption, the threat area was moving southwards, so re-routing around the north side of the threat area would be the better decision. Each experimental session included three simple and three complex decisions.

\section{Procedure}

After completion of training on the UAV supervision and replanning task and the Interruption Assistance Interface (if applicable), participants practiced performing and resuming the primary task until comfortable. Participants then performed the experimental trial for 35 minutes, in which they were interrupted six times from the primary task to complete a secondary task in the adjacent room. In the interruption task, participants were asked to spend roughly two minutes finding certain street and building locations using a simple online map tool. After primary task resumption, participants were asked to report all critical events that occurred during each interruption. Finally, participants completed a post-experiment questionnaire. The entire experimental session took approximately three hours.

\section{Data Collection and Measures}

All interactions with the primary task display and the Interface Assistance Interface were captured in a log file, which was used to determine participants' interruption recovery time, decision accuracy, and general interaction patterns. Event reports and pre- and post-experiment questionnaire data were collected from participants. Observer field notes were also collected to determine participants' interruption recovery strategies. Participants' decisions made following an interruption determined their decision accuracy score, which was determined as follows: $0=$ no replanning actions taken; 1 = replanning actions represented a suboptimal decision given available information; 2 = replanning actions corresponded to optimal decision.

\section{RESULTS AND DISCUSSION}

The data from this investigation suggest that both replay designs helped facilitate task resumption after an interruption of the UAV supervision and replanning task. The data also suggest that the interface design of an interruption recovery tool can impact the ability to access historical system information, thus an effective tool should be designed to minimize cognitive processing during task reorientation. These results are explained in more detail in the following sections.

\section{Task Resumption Performance}

Recovery time was similar across all the interruption recovery episodes, but the recovery time depended on the complexity of the situation faced after an interruption. The $3 \times 2$ repeated measures ANOVA for recovery time yielded non-significant results for the Assistance Type factor $(\mathrm{F}(2,7)=$ $.496, \mathrm{p}=.632, \alpha=.05)$ but significant results for Decision Difficulty $(F(1,6)=7.291, \mathrm{p}=.036)$. As Figure 5 shows, when faced with a complex decision participants tended to recover more quickly with any kind of assistance, particularly bookmarked assistance. In contrast, recovery time tended to be similar in both replay assistance conditions when faced with a simple decision.

Since recovery times only convey how fast operators responded, the number of events detected and the appropriateness of the responding participant actions were also measured. In general, the data show a trend of higher event detection when resuming the primary task after an interruption when participants were provided some type of replay assistance (with assistance: $90 \%$, no assistance: $75 \%$ ). Using the Kruskal Wallis test to compare decision accuracy in response to detected events across the different interfaces, for the simple decision factor, the assistive interfaces provided significantly more accurate decisions for the simple problems $(\chi 2=4.571, p=.102, \alpha=.1)$ but not for the complex Decision Difficulty factor $(\chi 2=.408, \mathrm{p}=.816)$ (Figure 6 ).

\section{Task Resumption Strategies}

Participants' interruption recovery strategies shed some light on the task resumption performance trends and on the impact of replay assistance. Participants with no assistance 
Cite as: Scott, S.D., Mercier, S., Cummings, M.L., \& Wang, E. (2006). Assisting Interruption Recovery in Supervisory Control of Multiple UAVs. In Proceedings of HFES 2006: 50th Annual Meeting of the Human Factors and Ergonomic Society, October 16-20, 2006, San Francisco, CA, USA.

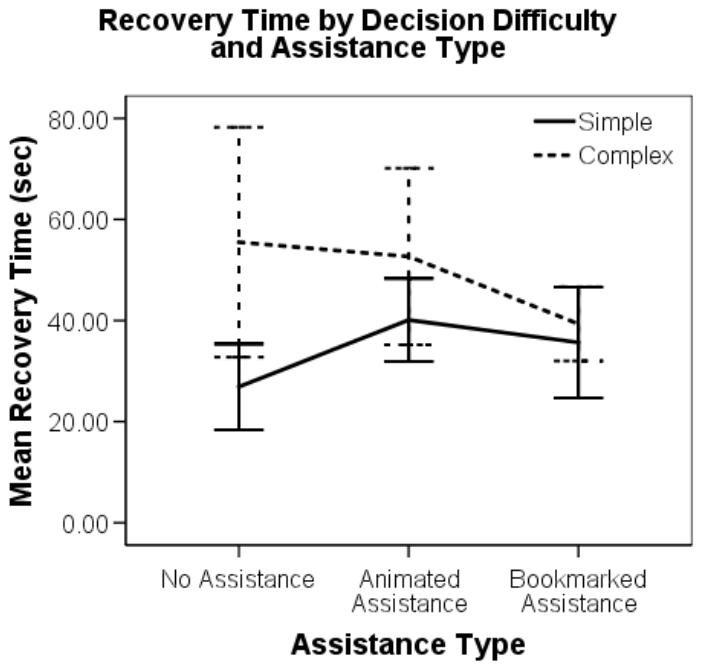

Figure 5. Mean recovery time by the difficulty of the decision faced after an interruption (complex or simple) by assistance type (no assistance, bookmarked assistance, or animated assistance).

typically looked at the map or the message history and took immediate action if the change in situation was obvious. In contrast, participants with replay assistance typically reviewed both the bookmarks and the message history before performing any replanning actions, possibly slowing their interruption recovery. However, their tendency to first review the bookmarks enabled them to notice complex events that they often further investigated in the replay window. Participants with no assistance had to learn of these events from the message history, demanding additional cognitive processing that contributed to more missed events and slower interruption recovery when faced with complex situation changes.

These recovery strategies also suggest that the event timeline on the assistive interfaces played an important role in the improved event detection and decision-making accuracy of participants with assistance when they faced simple situation changes. As mentioned above, these participants would typically first review the event timeline after each interruption. As illustrated in Figure 3, each event bookmark is displayed relatively positioned in time, providing a visual interpretation of the recency of each event. This display format simplified the process of detecting that an event had occurred during the interruption and, thus, required attention. Without this event timeline, participants in the no assistance condition had to first distinguish the emergent event from other messages in the message history and then mentally related the textual timestamp beside the event message to the current mission time.

When more complex situation changes occurred, participants with assistance were still more likely to detect events than participants without assistance. However, since decision accuracy decreased across all Assistance Type conditions, the event timeline, as well as the message history, appeared to be less useful for helping participants determine the correct response to complex events. Possible design
Decision Accuracy by Decision Difficulty and Assistance Type

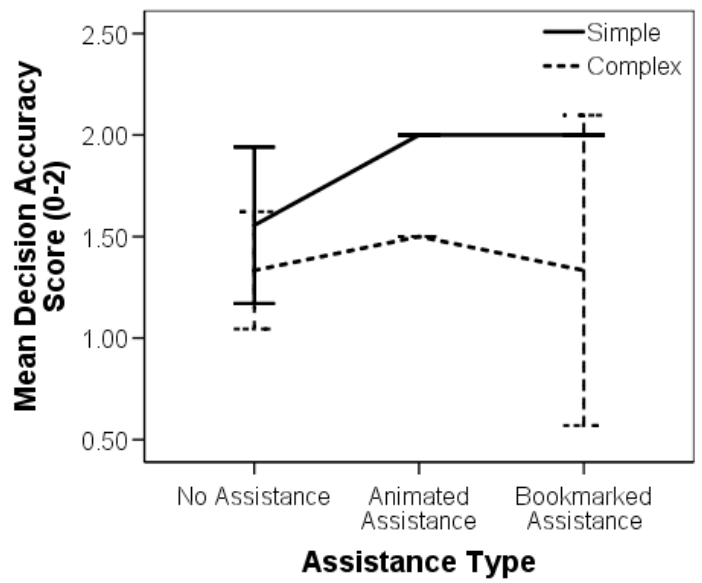

Figure 6. Mean decision accuracy score by the difficulty of the decision faced after an interruption (complex or simple) by assistance type (no assistance, bookmarked assistance, or animated assistance).

changes to the event timeline that might address this issue are discussed in the following section.

According to the observed participant task resumption strategies, the 'instant replay' feature was most often used when complex situation changes occurred. For these instances, the interruption recovery performance data showed a trend towards quicker recovery with bookmarked assistance as compared to animated assistance. This result, combined with participants' tendency to make equivalently accurate decisions with both assistive interfaces, suggest that participants with animated replay assistance spent more time interpreting the complex situation changes than participants with only the bookmarked assistance. This increased interpretation time was likely due to due to the time required to view system activity between events of interest (even at an accelerated rate), as compared to instantly jumping between events by clicking on different event bookmarks in the bookmarked assistance condition. It is not unexpected that the addition of a decision support tool will increase recovery times as compared to a system with no decision support. The design challenge, particularly for time-critical supervisory control systems, is to provide accurate decision support without incurring a significant time penalty.

\section{DESIGN IMPLICATIONS}

The observational data, along with participants' responses to the post-experiment questionnaire, suggest several important implications for the design of the Interruption Assistance Interface and interruption recovery tools for human supervisory control applications in general.

Enable User Control of Event Replay. Participants found the ability to select and view specific events (and when available, a particular sequence of events) very useful, especially for understanding complex system changes. Their comments indicated they desired even further control over the 
Cite as: Scott, S.D., Mercier, S., Cummings, M.L., \& Wang, E. (2006). Assisting Interruption Recovery in Supervisory Control of Multiple UAVs. In Proceedings of HFES 2006: 50th Annual Meeting of the Human Factors and Ergonomic Society, October 16-20, 2006, San Francisco, CA, USA.

interface, including the ability to drag the current time indicator on the elapsed time bar (Figure 3) anywhere on the timeline to view the system state.

Provide Visual Summary of Critical Events. The study findings suggest that providing a visual summary of past critical events with appropriate visual context can be particularly useful for identifying when complex system events have occurred in a human supervisory task. However, the findings also reveal that this visual summary can detract from task resumption when simple changes in system state have occurred, so careful design of this visual summary is essential, as discussed below.

Limit Visual Summary to Goal-Related Events. The trend of slower interruption recovery with an assistive interface after a simple system change suggests that the event timeline contained distracters that interfered with participants gleaning relevant event information, an issue confirmed by participant comments: several people found the event timeline cluttered and suggested including fewer bookmarks. The observational data revealed that participants relied heavily on the threat area and target event bookmarks before performing their replanning actions, only using the UAV status bookmarks to complete the event report. This behavior is not surprising given the mission criteria for the UAV supervision and replanning task: participants were responsible for striking targets on time and keeping the UAVs safe. Thus, the most useful bookmarks corresponded to events directly relevant to those mission goals, with all others appearing to be visual distracters from obtaining information relevant to these mission goals. Hence, to select the critical system events to include in a visual summary, the designer should consider those events most relevant to the mission or task goals and the corresponding system actions the user may perform during task resumption.

Clearly Indicate Relationships between Past and Current System State. The study findings revealed that one of the main limitations of our prototype replay interfaces was their lack of support for determining the relationship between the current system state and the past events indicated in the event timeline. One possible way to address this issue would be to integrate the two displays together, overlaying the current system state with the event history, representing the past events as 'trace' information. This issue needs further investigation though, since the impact of overlaying current information with the historical event information on interruption recovery is unclear.

\section{CONCLUSIONS}

This paper presents an initial investigation of the impact of enhanced video replay assistance on interruption recovery in a supervisory control task. These findings extend previous research by elucidating situations in which a replay interface would likely provide the most value to task resumption. Our results suggest that replay assistance can be particularly useful when participants are faced with complex system changes.
This work also builds on previous research by exploring new approaches for conveying and interacting with past system events. This investigation also provides several design recommendations for interruption recovery tools designed to support human supervisory control tasks.

Though this work helps extend the existing knowledge of interruption recovery tools for the human supervisory control application domain, additional work in this area is needed. In particular, further investigation is needed on how to mitigate the disadvantages of providing interruption assistance when only simple system changes have occurred. A better understanding of how to indicate the relationship between past and current system state, possibly through the integration of the replay and the main displays, is also needed.

\section{REFERENCES}

Altmann, E.M. \& Trafton, J.G. (2004). Task interruption: Resumption lag and the role of cues. In Proceedings of CogSci 2004: 26th Annual Conference of the Cognitive Science Society., Conference.

Bailey, B.P., Konstan, J.A. \& Carlis, J.V. (2001). The effects of interruptions on task performance, annoyance, and anxiety in the user interface. In Proceedings of INTERACT 2001: IFIP International Conference on Human-Computer Interaction, Conference, pp. 593601.

Cummings, M.L. \& Mitchell, P.J. (accepted). Automated Scheduling Decision Support for Supervisory Control of Multiple UAVs. AIAA Journal of Aerospace Computing, Information, and Communication.

Czerwinski, M., Cutrell, E. \& Horvitz, E. (2000). Instant messaging and interruption: Influence of task type on performance. In Proceedings of OZCHI 2000: Conference for the Computer-Human Interaction Special Interest Group of the Ergonomics Society of Australia, Conference, pp. 356-361.

Loukopoulos, L.D., Dismukes, R.K. \& Barshi, I. (2001). Cockpit interruptions and distractions: A line observation study. In Proceedings of thPe 11th International Symposium on Aviation sychology, Conference, Columbus, $\mathrm{OH}$, Ohio State University Press.

McFarlane, D.C. (1999). Coordinating the Interruption of People in Human-Computer Interaction. In Proceedings of INTERACT'99: 7th IFIP Conference on Human-Computer Interaction, Conference, Amsterdam, IOS Press, pp. 295-303.

St. John, M., Smallman, H.S. \& Manes, D.I. (2005). Recovery from Interruptions to a Dynamic Monitoring Task: the Beguiling Utility of Instant Replay. In Proceedings of HFES 2005: 49th Annual Meeting of the Human Factors and Ergonomics Society, Conference, Santa Monica, CA., Human Factors and Ergonomics Society, pp. 473-477. 